\title{
Drug Treatment of Disease
}

\section{DRUGS IN THE TREATMENT OF SYPHILIS：PART I*}

\author{
BY
}

A. J. KING, F.R.C.S.

Senior Physician, London Hospital Clinic for Venereal Diseases

\section{Toxicity of Penicillin}

Penicillin has deservedly enjoyed a reputation for lack of severity and infrequency of toxic effects, and this has given a degree of safety in the treatment of syphilis never before attained. Unfortunately, there is evidence of an increasing, although still small, incidence of hypersensitivity to the drug. It has been estimated (Feinberg and Feinberg, 1956) that reactions due to this cause develop in 1 to $5 \%$ of patients treated with penicillin. Reactions may be immediate or delayed. Immediate reactions take the form of acute anaphylaxis, which has been fatal in some cases. The delayed reaction appears from 24 hours to four weeks after administration of the drug and commonly takes a form resembling serum sickness. The manifestations include pruritus, urticaria, arthralgia, fever, and albuminuria. Exfoliative dermatitis and polyarteritis nodosa have also been described. Nearly all the patients who suffer reactions due to sensitization have received the drug before in some form, and most are aware of the fact. It is therefore an essential precaution to question patients about past treatment with penicillin, and reactions to it, before the drug is given. If there is a history of reaction due to sensitivity, treatment of this kind should not be given.

In cases of doubt, skin-testing may provide evidence of hypersensitivity, though not all patients who are sensitized give positive skin tests. A positive test should be accepted as a definite warning that a reaction is likely to follow administration of the drug. The most satisfactory test is probably an intradermal injection, and the dose may be $0.02 \mathrm{ml}$. of a solution containing 10,000 units of crystalline penicillin per $\mathrm{ml}$. If extreme sensitivity is suspected, it is probably wiser to perform a scratch test before giving the intradermal injection. False positive results are excluded by performing a similar test with the diluent or vehicle of the preparation of penicillin. A sensitized patient is likely to react to the scratch or intradermal test with weal-formation and erythema within 20 minutes. It has been suggested that the site of the skin test should be examined after 24 to 48 hours for delayed reaction, and that such reaction may indicate the possibility of a less severe sensitivity response to penicillin. Others have questioned the reliability of the delayed reaction. Smith (1957) recommended a simple test to detect liability to acute anaphylaxis. He applied drops of procaine penicillin $(300,000$ units per ml.) prepared for injection, to a skin scratch and to the conjunctival mucous membrane. If there was no reaction to either test within fifteen minutes, the injection was given. He claimed that this test indicated accurately patients liable to anaphylactic reactions. Positive tests were detected by such signs as itching, redness, oedema and weal formation of the skin, or itching, watering, redness, and oedema of the eye.

Treatment of acute anaphylaxis consists of intramuscular injection of a full dose of adrenaline (10 minims ( $0.6 \mathrm{ml}$.$) of a 1:1,000 solution) and, if necessary,$ intravenous administration of aminophylline $(250 \mathrm{mg}$. in $10 \mathrm{ml}$. of sterile water). Care should be taken to see that the patient's airway is unobstructed, and oxygen should be given if respiratory distress is acute. Artificial respiration may be necessary. Delayed reactions may be treated with antihistaminics or, if necessary, with corticotrophin or cortisone. A recent claim has been made (Minno and Davis, 1957) of useful results from the treatment of delayed reactions with penicillinase, given in a single intramuscular injection of 1 million units.

\section{Other Antibiotics}

Some other antibiotics have been shown to have an effect in the treatment of early syphilis, but, so far as the evidence goes, none can be regarded as an adequate substitute for penicillin. Turner and Schaeffer (1954) compared the antitreponemal effects of various antibiotics in rabbits infected with Treponema pallidum. The order of effectiveness appeared to be: (1) Penicillin; (2) carbomycin and erythromycin; (3) oxytetracycline and chlortetracycline ; (4) chloramphenicol and streptomycin. They suggested that carbomycin and, possibly, erythromycin appeared to be the most likely substitutes for penicillin against syphilis, and that they should receive clinical trial. But no other antibiotic at present available was considered to be in the same class as penicillin.

Clinical trial of antibiotics other than penicillin have been sporadic and inconclusive. The curative effect of streptomycin seems to be negligible. So slight is its effect that it has been used successfully as a substitute for penicillin in the treatment of gonorrhoea in cases in which there is a considerable likelihood that the patient is incubating syphilis, to prevent suppression of the early lesions with masking of the second and more important diagnosis.

Chloramphenicol by mouth has been shown to cause disappearance of Treponema pallidum from the infectious lesions of early syphilis in about 24 hours. This was followed by satisfactory healing of lesions and, later, reversal of positive serological tests to negative. Dosage has been from 40 to $100 \mathrm{mg}$. per $\mathrm{kg}$. of body weight, daily for six to eight days, or, in one series, $1 \mathrm{~g}$. by mouth every six hours to a total of $40 \mathrm{~g}$. One small group of 13 patients (Robinson and Robinson, 1953) was observed for two to four years after treatment of this kind. Five were apparently cured; three had relapsed; and serological tests remained positive in three cases and doubtfully positive in two. All 13 had negative tests of the cerebrospinal fluid. Toxic effects of the drug were limited to diarrhoea, papular eruptions, and stomatitis. There was no reported case of blood dyscrasia.

Chlortetracycline by mouth gave satisfactory immediate effects in the treatment of cases of infectious syphilis. The organism disappeared from surface lesions in from two to five days. Lesions healed rather more 
slowly than with penicillin or organic arsenicals. Serological tests became negative in most cases. Dosage, in one series, was $2 \mathrm{~g}$. initially, followed by $2 \mathrm{~g}$. four hours later, and then $1 \mathrm{~g}$. every four hours, day and night, until $70 \mathrm{~g}$. had been given in 11 days. In another series dosage of $60 \mathrm{mg}$. per $\mathrm{kg}$. body weight per day for eight days seemed satisfactory. Rodriquez et al. (1952), who used the former scheme of treatment, reported on 101 patients so treated and observed for 12 to 15 months. Treatment seems to have been successful in about $75 \%$.

Oxytetracycline by mouth has given results similar to those described with chlortetracycline. Dosage in one small series was $3 \mathrm{~g}$. by mouth followed by $0.5 \mathrm{~g}$. every four hours for 15 days, giving a total of $48 \mathrm{~g}$. (Robinson and Robinson, 1951). Treponemes disappeared in 4 to 48 hours. Observation was possible in four cases and all became sero-negative with later sero-relapse in one case. The same authors (1953) examined the three remaining patients two to four years after treatment. All were well with negative serological tests and tests of the cerebrospinal fluid. Irgang and Alexander (1953) treated 10 cases of early syphilis with oxytetracycline by mouth, giving $2 \mathrm{~g}$. a day for 14 days. Treponema pallidum disappeared from surface lesions in 44 to 96 hours. In most cases the surface lesions responded favourably, but in a few instances they were slow to resolve. Serological response was favourable in those patients who remained under observation. One case proved resistant to treatment. Baler (1956) gave oxytetracycline intramuscularly in the treatment of 16 patients suffering from early syphilis. Dosage was 200 mg. twice daily for 10 days, the total being $4 \mathrm{~g}$., except in one case in which it was $100 \mathrm{mg}$. twice daily for three days, followed by $200 \mathrm{mg}$. twice daily for seven days (3.4 g.). All lesions healed rapidly and Treponema pallidum disappeared in an average of 40 hours. The treatment failed in four cases, but was satisfactory in the others after 8 to 16 months. One patient with secondary syphilis was delivered of a full-term healthy infant 119 days after the start of treatment.

As to carbomycin, Buckinger et al. (1955) treated 11 patients suffering from early syphilis with this preparation by mouth, giving 2 to $3 \mathrm{~g}$. daily. Treponema pallidum disappeared from the lesions in 36 to 72 hours after the initial dose. Healing of lesions was satisfactory and toxic effects were slight. This was a preliminary and inconclusive report which appears not yet to have been confirmed.

Thus, if a patient sensitized to penicillin develops syphilis, the most reasonable course of action seems to be to administer oxytetracycline or chlortetracycline in the dosage described. It has been suggested that such patients may be treated with penicillin by suppressing reactions with the concurrent administration of corticotrophin. Such a procedure is obviously fraught with danger, and, if it is to be attempted at all, should be done in hospital under close supervision.

\section{REPERENCES}

Baler, G. R. (1956). Arch. Derm. Syph. (Chicago), 73, 489. Buckinger R H., Hookings, C E., and Garson, W. (1955). Antibiot. Med., I. 100

Buckwalter. F. H.. and Dickison, H. L. (1948). J. Amer. Pharm. Ass., sci. Ed.. 37, 472.

Feinherg. S. M, and Feinberg, A. R. (1956). J. Amer. med. Ass. 160. 778

Irgang. S., and Alexander, E R. (1953). Amer. J. Svph., 37, 247.

McFlligott. G. L. M. (1954). Brit. J. vener. Dis., 30. 17.

Minno. A. M., and Davis, G. M. (1957). J. Amer. med. Ass., 165 222.
O'Brien. J. F., and Smith, C. A. (1952). Amer. J. Syph., 36, 519. Robinson, H.M., and Robinson, H. M. (1951). Ibid., 35, 479. Robinson, (1953). Ibid., 37.243 .

Rodriquez, J., Weinstein, S., and Parkhurst, G. E. (1952). Arch. Derm. Syph. (Chicago), 66, 59

Smith, V. M. (1957). New Engl. J. Med., 257, 447

Szabo, J. L., Edwards, C. D., and Bruce, W. F. (1951). Antibiot. and Chemother., 1, 499.

Turner, T. B., and Schaeffer, K. (1954). Amer. J. Syph., 38, 81.

\section{USE OF SURGICAL REHABILITATION IN YOUNG DELINQUENTS*}

\author{
BY
}

D. A. OGDEN, L.M.S.S.A.

Medical Officer, H.M. Prison Medical Service

As a result of the physical and psychiatric examination of several thousand Borstal inmates over a period of years, certain impressions become quite clear, and one of these is a sense of neglect. This neglect is now widely accepted by most workers in criminology to be basically one of lack of moral values and practical example in the family circle. As a result of these examinations it became evident that in many cases this neglect spreads also into physical matters. Thus squints, nasal deformities, and other varied disabilities are left uncorrected in childhood and allowed to become either foci of resentment or of chronic irritation.

In 1950, at Camp Hill Borstal Institution, a brief pilot series of selected cases was submitted for surgical opinion and treatment, and the results appeared encouraging enough to suggest that a more detailed study should be carried out with the express purpose of determining whether surgical rehabilitation, in removing these defects, could also produce secondary psychological effects which would be of use in combating delinquency. This project was put into action during 1951 to 1955 at H.M. Borstal Institution, Portland, and is the subject of this paper.

\section{Present Investigation}

It was soon clear that such a project demanded the interest and support of the various surgeons who would be concerned with the work, and some time was spent in personal explanations. The co-operation given by the surgeons in the area is greatly appreciated. Similarly, appreciation is due to the Dorset Hospital Management Committee.

The next question to be settled was the timing of the treatment. In normal medical practice it would be reasonable to expect individuals to seek assistance when troubled by a disfigurement, but to rely on this in a young delinquent population would be unrealistic. The very sense of neglect and, in many cases, rejection felt in the home circle has usually long since buried any hope that something may be done. Accordingly it is necessary for the doctor to raise the question of rehabilitation and not the patient. Furthermore. it is not reasonable to request undue priority for remedial cases competing for hospital bed accommodation with other surgical cases, and therefore it is expedient to start action early enough to allow time on waiting-lists.

It was therefore concluded that the question of possible remedial action should be raised at the original

*Published by permission of H.M. Prison Commissioners, though it does not necessarily represent their views. 